CASO CLÍNICO

\title{
Diagnóstico de policitemia absoluta como posible causa de episodios convulsivos en un perro
}

\section{Diagnosis of absolute polycythemia as possible causes of seizures in a dog}

\author{
Sandra Acevedo $\mathrm{T}^{1 *}$ Esp, Mauricio Ramírez L, ${ }^{1}$ Esp. \\ ${ }^{1}$ Universidad de Antioquia, Facultad de Ciencias Agrarias, Grupo de investigación CENTAURO. Medellín, \\ Colombia.*Correspondencia: crisalida18@agronica.udea.edu.co
}

Recibido: Septiembre 16 de 2009; Aceptado: Julio 28 de 2010.

\section{RESUMEN}

Se expone el caso de un perro poodle de 3 años de edad, el cual hace un año comenzó a convulsionar esporádicamente, principalmente después de hacer ejercicio y en algunas ocasiones presentó temblor generalizado. Al examen clínico realizado se observó mucosas orales y oculares hiperémicas. Al realizar los exámenes paraclínicos complementarios se encontró en hemogramas seriados un aumento marcado del hematocrito y la hemoglobina con niveles de proteínas plasmáticas en rangos normales, y una eritropoyectina 10 veces por debajo del rango normal. Se realizó ecografía abdominal y evaluación cardiaca. El diagnóstico fue de policitemia absoluta como la causa de las convulsiones.

Palabras clave: Canino, convulsión, eritropoyetina, hematocrito, policitemia.

\section{ABSTRACT}

The case of a 3-year-old poodle, which last year began to suffer sporadic seizures, especially after exercise and sometimes-presented generalized tremor, is reported. At clinical examination the hyperemic eye and oral mucosa were observed. Additional Laboratory tests performed showed that serial blood counts increasingly marked the hematocrit and hemoglobin with levels of plasmatic proteins within normal ranges, and erythropoietin was 10 times under the normal range. An abdominal ultrasound and a cardiac evaluation were performed. The diagnosis was absolute polycythemia as the cause of seizures.

key words: Policitemia, eritopoyectina, hematocrito, convultion, canine 


\section{INTRODUCCIÓN}

La policitemia se define como el hallazgo de un recuento celular elevado de glóbulos rojos, se expresa como un aumento del volumen celular aglomerado (VCA) por encima de los rangos de referencia, siendo esta lo contrario a un proceso anémico. (1-3). En medicina veterinaria otras líneas celulares sanguíneas no necesariamente se ven involucradas, mientras que cuando esta enfermedad se presenta en humanos se comprometen otras líneas como las plaquetas y en ocasiones los glóbulos blancos. $(1,4,5)$.La policitemia se puede presentar en pacientes con ciertos tipos de tumores vasculares como leihomiomas y hemangiosarcomas los cuales llevan a una sobreproducción de eritopoyectina por hipoxia renal (6-8) entre otras causas de la eritocitosis se encuentran los shunts arteriovenosos, deshidratación severa, hiperadrenocortisismo, policitemia vera (policitemia primaria) y en general enfermedades crónicas de tipo cardiaco y pulmonar $(9,10)$.

La policitemia se clasifica como relativa o absoluta según la etiología $(1,3,11)$. La relativa se refiere a hemoconcentración; la masa celular de glóbulos rojos se encuentra normal pero aparentemente estos están incrementados de tamaño es decir no se produce aumento real de la masa eritrocitaria $(4,11,12)$. La deshidratación es la causa mas común de policitemia relativa en pacientes caninos $(3,13)$, en donde el VCA, el hematocrito y la concentración de proteínas plasmáticas están leve o severamente incrementados debido a que existe una eventual disminución en el fluido corporal total (14-16). Su diagnostico se basa en valorar la apariencia clínica del paciente, el cuadro hematico completo y en el análisis e interpretación de pruebas paraclinicas sanguíneas, como la concentración de nitrógeno ureico sanguíneo (BUN) y creatinina que pueden estar incrementadas así como la densidad urinaria la cual generalmente se encuentra concentrada o hiperestenurica $(1,7,8)$. La adrenalina e histamina liberada por animales asustados o sometidos a factores de stres causa una contracción esplénica y redistribución de las células sanguíneas, pero esta forma de policitemia es pasajera y no está asociada a incremento en la concentración de proteínas plasmáticas. $(11,17,18)$

La policitemia absoluta o vera es un desorden mieloproliferativo $(4,9,16)$ donde el defecto podría estar a nivel de las células multipotenciales o en el sistema hematopoyetico, generándose un aumento en la masa celular de glóbulos rojos, hemoglobina, recuento de glóbulos rojos y hematocrito con una concentración normal de proteínas plasmáticas $(6,12,19)$. Se conoce que esta se presenta con mayor frecuencia en perros de mediana edad y no hay predilección por raza especifica $(1,3,17)$.

Los síntomas de la policitemia vera, dependen del grado de viscosidad de la sangre pudiéndose formar trombos disminuyendo la microcirculación provocando hipoxia local, causando signos de tipo neurológicos como convulsiones, ataxia, ceguera, temblores o cambios de comportamiento. $(1,15)$

En el presente artículo se describe el caso de un paciente canino el cual presentaba convulsiones aisladas que en los últimas semanas aumentaron en intensidad y frecuencia. En los exámenes complementarios realizados la única anormalidad encontrada fue una policitemia y al profundizar con diversas ayudas paraclinicas se diagnostica una policitemia primaria se discute su diagnostico, tratamiento y evolución. Al descartar las demás etiologías causantes de convulsión se llega al diagnostico presuntivo de policitemia causante de las convulsiones.

\section{Presentación del caso}

Reseña. Perro de raza poodle macho de 3 años de edad, peso de $5.8 \mathrm{Kg}$. con plan de vacunación y desparasitación vigente, el cual desde hace un año presentaba episodios esporádicos convulsivos no tratados, pero que en las últimas 3 semanas han aumentado en intensidad y frecuencia presentándose estos después de realizar ejercicio o ante un evento que genere excitación en el paciente.

Anamnesis. Los propietarios reportan que desde hace un año ha sufrido episodios 
convulsivos esporádicos, pero que desde hace algunas semanas estos episodios se han hecho más frecuentes e intensos, principalmente después de realizar ejercicio, y en algunas ocasiones en momentos de excitabilidad, como cuando llega uno de los propietarios.

Hallazgos al examen físico. Paciente atento al medio pero con marcada ansiedad. Mucosa ocular y oral hiperémicas, jadeo, taquicardia, pulso fuerte sin signos de deshidratación (Tabla 1). A la auscultación cardiaca, pulmonar y palpación abdominal no se hallaron anormalidades. El examen nueorológico fue normal.

Tabla 1. Constantes fisiológicas del paciente en la evaluación inicial.

\begin{tabular}{lcc}
\hline \multicolumn{1}{c}{ Parámetro } & Valor & Valores de referencia \\
\hline Temperatura corporal & $38.2 \mathrm{C}^{\circ}$ & $38.5-39.5 \mathrm{C}^{\circ}$ \\
Frecuencia cardiaca & $166 \mathrm{lat} / \mathrm{min}$ & $80-100 \mathrm{lat} / \mathrm{min}$ \\
Frecuencia respiratoria & $28 \mathrm{r} / \mathrm{min}$ & $10-30 \mathrm{~min}$ \\
Peso & $6 \mathrm{Kg}$ & \\
Apariencia general & anormal (estado de confusión) \\
Palpación abdominal & & normal \\
\hline
\end{tabular}

Ayudas diagnósticas. Se realizó hemograma, ALT, creatinina, Ca, eritropoyetina, T4 y glicemia, tratando de descartar una causa no neurogénica o extracraneal de las convulsiones presentadas por el paciente $(1,8)$. Los resultados de las pruebas químicas se encontraron dentro de los parámetros normales. El hemograma reveló un aumento del hematocrito y de la hemoglobina de $56 \%$ y $18.1 \mathrm{~g} / \mathrm{dl}$ respectivamente y con proteínas totales en $6.6 \mathrm{~g} / \mathrm{dl}$. (Tabla 2). Los resultados de eritropoyetina fueron los siguientes: $1.0 \mathrm{u} / \mathrm{ml}$ siendo el rango normal de $88.2 \mathrm{u} / \mathrm{ml}$. Correspondiendo esto con un cuadro hemático compatible con policitemia $(12,15)$ frente a lo cual se determinó no iniciar una terapia médica anticonvulsiva hasta encontrar la causa real del problema. Previo a los exámenes paraclínicos se realizó ecografía abdominal, ecocardiografía y placas radiográficas de tórax y abdomen descartando presencia de tumores o enfermedad cardiaca o pulmonar concomitante.
Tabla 2. Resultados del hemoleucograma \#1

\begin{tabular}{lccc}
\hline \multicolumn{1}{c}{ Parámetro } & $\mathbf{U}$ & Valor & $\begin{array}{c}\text { Valores de } \\
\text { referencia }\end{array}$ \\
\hline Hematocrito & $\%$ & 55.7 & $35.2-52.8$ \\
Hemoglobina & $\%$ & 18.1 & $12.7-16.3$ \\
Eritrocitos & Eri/ul & $8^{\prime} 100.000$ & $\begin{array}{c}5.3^{\prime} 000.000- \\
8^{\prime} 600.000\end{array}$ \\
Leucocitos & Leu/ul & 8.100 & $8.300-17.500$ \\
Neutrófilos & $\%$ & 75 & $65-73$ \\
Eosinófilos & $\%$ & 3 & $1-8$ \\
Linfocitos & $\%$ & 37 & $9-26$ \\
Plaquetas & $\mathrm{Plt} / \mathrm{ul}$ & 238.000 & $160.000-$ \\
Proteinas totales & $\mathrm{g} / \mathrm{dL}$ & 6.6 & $6.0-8.0$ \\
\hline
\end{tabular}

U: Unidad

Ecocardiografía. Modo B-Doppler Color: Ventana para esternal derecha: Eje Largo; Se aprecia el ventrículo y atrio izquierdo con tamaño cameral adecuado para la talla; válvula mitral de anatomía y cierre adecuado, no hay regurgitación; septo interventricular de tamaño normal e integro; ventrículo y atrio derecho normal. Válvula tricúspide de anatomía y cierre adecuado, no hay regurgitación. No hay presencia de masas o trombos intracavitarios. No hay presencia de masas pericardiaco. En conclusión, ecocardiografía normal.

Interpretación radiográfica. En la proyección LL y DV de tórax y abdomen, no se apreciaron cambios en la estructura cardiaca ni en campos pulmonares, así como tampoco en cavidad abdominal. No se observaron masas ocupantes ni en tórax, ni abdomen.

Enfocándose básicamente en confirmar la policitemia vera como causa primaria de las convulsiones, se realizaron otros 2 hemogramas de control a los 8 y 15 días respectivamente, recomendándole a los propietarios del paciente quietud absoluta previa a la toma de la muestra, para evitar alteraciones en al cuadro hematico debido al ejercicio $(4,12,20)$. En los hemogramas se encontró un aumento leve en el hematocrito y en la hemoglobina con niveles de proteínas plasmáticas sostenidos (Tabla 3). Por lo anterior se decidió realizar una flebotomía como método diagnóstico y terapéutico $(1,3,7)$. Este procedimiento se realizó según 
Tabla 3. Resultados del hemoleucograma \#2

\begin{tabular}{lccc}
\hline \multicolumn{1}{c}{ Parámetro } & $\mathbf{U}$ & Valor & $\begin{array}{c}\text { Valores de } \\
\text { referencia }\end{array}$ \\
\hline Hematocrito & $\%$ & 56.2 & $35.2-52.8$ \\
Hemoglobina & $\%$ & 18.4 & $12.7-16.3$ \\
Eritrocitos & Eri/ul & $7^{\prime} 570.000$ & $\begin{array}{l}5.3^{\prime} 000.000 \\
-8^{\prime} 600.000\end{array}$ \\
Leucocitos & Leu/ul & 12.100 & $8.300-$ \\
& $\%$ & 75 & $65-73$ \\
Neutrófilos & $\%$ & 3 & $1-8$ \\
Eosinófilos & $\%$ & 20 & $9-26$ \\
Linfocitos & $\%$ & & $160.000-$ \\
Plaquetas & $\mathrm{Plt} / \mathrm{ul}$ & 235.000 & 525.000 \\
Proteinas totales & $\mathrm{g} / \mathrm{dL}$ & 7.0 & $6.0-8.0$ \\
\hline U: Unidad & & &
\end{tabular}

el protocolo recomendado por Kienle (9). El paciente presentó un hematocrito de $56 \%$ y se calculó uno deseado del $50 \%$ a fin de evitar efecto rebote, según la formula:

Sangre a remover $(\mathrm{ml})=[$ (peso del paciente $\mathrm{kg} \times 0.08) \times 1000 \mathrm{ml} \times$ (hto actual - hto deseado) / Hematocrito actual]

Se extrajeron $52 \mathrm{ml}$ de sangre los cuales se restituyeron a través de fluidos endovenosos Hartman 2 veces el volumen obtenido de sangre o sea $188 \mathrm{ml}$ en 3 horas. En donde efectivamente el hematocrito y la hemoglobina disminuyeron. Se midieron niveles sericos de eritropoyetina $(4,6,7)$ encontrando un valor con una marcada disminución por debajo del promedio reportado en caninos (Resultado de la prueba $1.0 \mathrm{u} / \mathrm{ml}$ y rango normal $88.2 \mathrm{u} / \mathrm{ml}$ ) lo cual confirma el diagnóstico de policitemia absoluta.

Este nivel se encuentra muy por debajo de los niveles normales debido a que como el paciente presenta un severo aumento en la línea roja la hipófisis realiza una retroalimentación negativa causando a nivel de las células yuxtaglomerulares una disminución en la producción de esta hormona $(4,7)$.

Debido a que todo orientaba a un diagnostico de policitemia vera se decidió descartar otras posibles causas de eritrocitosis como masas neoplásicas ocupantes y cardiopatías $(12,13,18)$ realizando al paciente radiografía
LL y DV de tórax, ecografía abdominal, ecocardiografia y electrocardiograma descartando con estas cualquiera de las patologías antes mencionadas.

Tratamiento. Durante el tiempo de diagnostico el paciente solo presentó un episodio convulsivo de intensidad moderada, luego de este se realizó la primera flebotomía, por medio de esta se logró controlar los episodios convulsivos del paciente. Se recomendó al propietario manejar hidroxiurea, medicamento recomendado en estos casos ya que inhibe la enzima ribonucleósidodifosfato - reductasa logrando causar una mielodepresión en los pacientes $(4,7,15)$ acompañada de las flebotomías pero por los posibles efectos secundarios de este medicamento, cursando desde leucemogénesis como el más grave, hasta anorexia, vómito e hiperpigmentación, el escalamiento, el eritema, la alopecia, la descamación de la región facial $(8,15,18)$ y la manipulación necesaria del propietario del paciente no accedió a instaurar dicho tratamiento.

Cuatro meses después de la flebotomía el paciente nuevamente comenzó a presentar convulsiones agrupadas, por ello se decidió realizar otra flebotomía bajo las condiciones antes mencionadas, en donde se obtuvo muy buena respuesta en el control convulsivo. Sin embargo al cabo de 3 meses el paciente nuevamente comenzó con episodios convulsivos, pero los propietarios no autorizaron el tratamiento.

\section{DISCUSIÓN}

La policitemia absoluta es un diagnostico diferencial de convulsiones en un caninos (8), que puede desencadenar episodios que cursan desde muy esporádicos hasta frecuentes y, si no es tratada correctamente puede causar afecciones mucho más graves 0 inclusive hasta la muerte $(3,4,6)$. Entre los daños o alteraciones sistémicas se describen entre otros, disnea de moderada a severa y cianosis marcada generalmente en las extremidades por la evidente hemoconcentración o la viscosidad $(1,18)$. En este caso el paciente no presentó ningún 
síntoma de tipo cardiovascular o vascular periférico como se descartó debidamente por medio del examen físico y de las pruebas realizadas para este efecto; por lo tanto se puede inferir que las anormalidades físicas no cumplen un orden característico en su presentación y que aunque se esperaría que el paciente, al presentar signología de tipo nervioso debería cursar antes con signología cardiovascular esto no sucedió, dando clara muestra que en este caso la policitemia primaria no necesariamente cursa con alteraciones vasculares periféricas como inicio de la gravedad del problema, sino que en esta es posible la presentación de cualquier otra enfermedad concomitante y que no existe un orden especifico para su presentación, en donde se esperarían alteraciones periféricas antes de alteraciones mucho mas graves cursando desde cianosis en las extremidades antes que alteraciones de tipo anóxico cerebrales que causen convulsiones como en este caso se expone.

No se realizó el examen sistémico de la medula ósea, ya que es un método altamente invasivo y no permite distinguir entre policitemia primaria y secundaria. En cualquier caso el componente eritroidemiloide es normal o esta disminuido (1).

Las flebotomías son el principal método terapéutico para el tratamiento de policitemia primaria $(3,11,14)$ y mediante este método es posible lograr una sobrevida de los pacientes de hasta 6 años (1). En este caso y como se mencionó antes, se realizaron dos flebotomías con intervalos de 4 meses mediante las cuales se logró interrumpir satisfactoriamente los episodios convulsivos.

Existen reportes de tratamientos por medio de estas con resultados muy satisfactorios. $(1,15,19)$. En el caso expuesto la indicación era el tratamiento con flebotomías seriadas teniendo en cuenta la adecuada respuesta del paciente y que por medio de estas se logró interrumpir los episodios convulsivos. Aunque la indicación era esa y teniendo en cuenta los resultados, se planteó la posibilidad de continuar con las flebotomías, pero por cuestiones culturales es difícil para los propietarios autorizar el tratamiento y someter a su mascota a este procedimiento por la manipulación y por los costos que esta puede implicar.

La hidroxiurea como medicamento mielodepresor es la herramienta básica junto con las flebotomías para el control y manejo de la policitemia primaria $(5,11,20)$. Este medicamento presenta una respuesta adecuada causando una mielosupresión que si es controlada y manejada presenta una mejor recuperación y mantenimiento para el paciente. Los efectos secundarios reportados con el uso de este medicamento como: anorexia, vómito, hiperpigmentación, escalamiento y descamación de la región facial, así como la falta de experiencia de los autores para su manejo y los pocos reportes que se encuentran acerca del uso de este en medicina veterinaria, hacen que su terapéutica sea difícil. Por lo tanto, como indicación médica presenta serias precauciones que no permitieron su manejo en el caso expuesto, debido a que se hace complicado su uso en pacientes caninos.

Para este caso se pretendió hacer con el objetivo de tratar de mantener niveles adecuados del hematocrito, la hemoglobina y un control de los demás factores sanguíneos, los cuales probablemente serian seriamente afectados y alterados causando una descompensación hematológica y sistémica del paciente, por lo que no se instauró tratamiento con la hidroxiurea aunque fuera la terapia de elección, quedando su manejo, terapéutica e indicación para un estudio posterior en el que se controlen y reporten los riesgos que este medicamento pueda causar en los pacientes.

El diagnóstico diferencial de la epilepsia con otros trastornos neurológicos es imprescindible, a fin de establecer el diagnóstico exacto y excluir crisis nerviosas como los desvanecimientos, sincopes o crisis por anormalidades reversibles como hipoglucemia, hipocalcemia, policitemia, fármacos, entre otros $(1,3,16)$. En casos como el del presente informe, aunque el diagnostico de los episodios epilépticos convulsivos fue concluyente y no necesariamente fue a causa de factores neurológicos sino sistémicos como anteriormente se describió, su tratamiento y 
manejo en un principio fue el adecuado por medio de las flebotomías con los resultados anteriormente expuestos.

Cabe anotar que a los propietarios del paciente se les brindaron múltiples opciones con respecto al manejo y adecuada terapia según el diagnostico presentado pero estos de ninguna manera aceptaron las sugerencias y opciones brindadas.

La medición de los niveles séricos de la eritropoyectina es la prueba fundamental en el diagnóstico de la policitemia primaria $(4,21)$. Esta prueba confirma la afección a nivel mieloproliferativa como un desorden a nivel del tallo clonal $(4,9)$, actuando como un regulador químico hormonal el cual es detectado a nivel central y causa una retroalimentación negativa con un resultante descenso de sus niveles para tratar de sostener un hematocrito normal $(1,13,16)$.

Por lo anterior, entre mayor sea la hemoconcentración, menores son los niveles de esta hormona, puesto que responde a una supresión causal de las células yuxtaglomerulares, para tratar de mantener un control en el hematocrito, hemoglobina y plaquetas $(4,19,10)$ lo cual sucedió en el caso que se expone, en donde los niveles de esta hormona se encontraron 10 veces por debajo de los niveles normales de un canino situación que aclara el diagnostico de este paciente, concluyendo que las convulsiones correspondían a esta patología y esta a su vez no era causa subsecuente de otra anormalidad sistémica, sino que la policitemia vera desencadenó ciertos desordenes sistémicos como las convulsiones.

En conclusión, los resultados del caso clínico demuestran que existen varias alteraciones de tipo sistémico que causan desordenes neurológicas convulsivo, es de suma importancia el conocimiento, valoración y diagnostico preciso de estas anormalidades.

En ciertas ocasiones los pacientes con estas afecciones pueden ser sujeto de un subdiagnóstico y por ende de un manejo médico y terapéutico que generalmente no es el adecuado, puesto que si no se corrige el factor primario, no es posible el manejo correcto y eficaz del problema.

\section{REFERENCIAS}

1. Andrea HH. Policitemia. Textbook of veterinary internal medicine. 6 a ed. St. Louis: Saunders; Ettinger S], Feldman CE (eds): 2005.

2. Ceballos AM. Generalidades sobre hematología. Manizales. 2004. 1-15.

3. Jill EM, Stephen P. Farmacología clínica en pequeños animales. Argentina. 2004. 284-287.

4. Mave Gm. Pathology domestic animals. 5 Ed. New York. 2007. 210-215.

5. Woll' en $\mathrm{NH}$, Ladjevardi M, Albert J, et al. Influence of different anticoagulants on platelet aggregation in whole blood: A comparison between citrate, low molecular mass heparin and hirudin. Thromb Research 1997;87:151-157.
6. Cowell RL, Tyler RD, Meinkoth $\mathrm{JH}$. Diagnostic cytology and hematology of the dog and cat. $3^{\text {rd }}$ ed. Canada; 2008. 399-402.

7. Felman ED, Nelson R. Endocrinología y reproducción en perros y gatos. $2^{\mathrm{a}}$ ed. Mexico; 2000. 564-568.

8. Lorenzo VF, Bernandini M. Neurología del perro y del gato. Argentina 2007. 347-352.

9. Kittelson MD, Kienle RD. Small animal cardiovascular medicine. St. louis: Mosby; 1998. 228-229.

10. Wilthrow ST, Vail D. Small animal Clinical Oncology. 4a ed. Canada; 2007. 84: 760-766. 
11. Guido F, Tiziano B. How I treat patients with polycythemia vera. The American Society of Hematology. 2008; 104-111. (Abstract).

12. Brener W, Darbes J, Hermmanas W. Idiophatic Mielofibrosis in a Cat and in three dog. 1999. J. Comparative Hematology International.1999; 1-8.

13. Nelson RW, Couto GC. Medicina Interna de animales pequeños. 3rd ed. Buenoa aires. 2005. 1243-1245.

14. Rebar AH, Mae P, Fred IM. Manual de hematología de perros y gatos. Barcelona. 2002. 68-71

15. Irimajiri M, Jay EE, Larry $T$. Mild Policitemia associated with compulsive disorder in dogs. Chicago. 2006. 23-28.

16. Willard $M$, Tvedten $H$. Small animal clinical diagnosis by laboratory methods. $4 a$ ed. Philadelphia: Saunders-Elservier. 2004: 412-118.
17. Valli VE. Veterinary Comparative Hematopathology. Australia. 2007. 440-443.

18. Briere J. Essential thombocythemia. 2007. J Orphanet of Rare Diseases. 2007; 1-17. (Abstract).

19. Woll 'en $\mathrm{NH}$, Ladjevardi $\mathrm{M}$, Albert J, et al. Influence of different anticoagulants on platelet aggregation in whole blood: A comparison between citrate, low molecular mass heparin and hirudin. Thromb Research 1997; 87:151-157.

20. Robert $\mathrm{H}$, Dunlop $\mathrm{CH}$. Veterinary Pathophysiology. Australia 2004. 504-508.

21. Woll'en $\mathrm{NH}$, Held $\mathrm{C}$, Rehnqvist $\mathrm{N}$, et al. Effects of mentaland phycias stress on platelet function in patients with stable angina pectoris and healthy controls. Eur Heart J 1997;18:807-815. 